\title{
VALORES DE REFERÊNCIA DE INGESTÃO DE NUTRIENTES PARA AVALIAÇÃO E PLANEJAMENTO DE DIETAS DE CRIANÇAS DE UM A OITO ANOS
}

\author{
DIETARY REFERENCE INTAKES FOR THE EVALUATION AND PLANNING OF \\ DIETS FOR CHILDREN AGED ONE TO EIGHT YEARS
}

Marta N. C. M. Vieira ${ }^{1}$, Camila C. Japur², Cristina M. M. Resende ${ }^{3}$, Jacqueline P. Monteiro ${ }^{1}$

1Docente, ${ }^{2}$ Nutricionista, Curso de Nutrição e Metabolismo da Faculdade de Medicina de Ribeirão Preto - USP. ${ }^{3}$ Mestranda, ${ }^{1},{ }^{3}$ Departamento de Pediatria e Puericultura da Faculdade de Medicina de Ribeirão Preto - USP.

Correspondência: Profa.Dra. Marta N. C. Marçal Vieira. Rua das Paineiras, 5. Campus USP. CEP: $14049-900$ - Ribeirão Preto / SP. Telefone: (16)3602-3095 / Fax:(16) 3602-3098. marta@fmrp.usp.br, camila@fmrp.usp.br, cmmresende@ig.com.br, jacque@fmrp.usp.br

Vieira MNCM, Japur CC, Resende CMM, Monteiro JP. Valores de referência de ingestão de nutrientes para avaliação e planejamento de dietas de crianças de um a oito anos. Medicina (Ribeirão Preto) 2008; 41 (1): 67-76.

RESUMO: Os valores de referência de ingestão dietética (Dietary Reference Intakes - DRIs) foram desenvolvidos para auxiliar no planejamento alimentar e avaliação da ingestão de nutrientes de indivíduos e populações. Foram propostos a partir do final da década de 90, em substituição às recomendações estabelecidas em 1989 (RDAs), visando a implementação de inovações como a criação de novos conceitos de avaliação e planejamento de dietas, devido à disponibilidade de informações atualizadas sobre necessidades e ingestão de nutrientes; o estabelecimento dos níveis máximos de ingestão com o surgimento do conceito de redução do risco de doenças crônico-degenerativas e a subdivisão dentro de cada faixa etária, devido às demandas diferenciadas de nutrientes para cada etapa da vida. Considerando a importância da avaliação do consumo alimentar na atenção integral à saúde da criança e a fim de subsidiar os profissionais de saúde no diagnóstico e acompanhamento nutricional, o objetivo deste trabalho foi apresentar de maneira prática as recomendações nutricionais e seu modo de aplicação em crianças de um a oito anos de idade baseando-se na metodologia das DRIs.

Descritores: Nutrição Infantil. Necessidades Nutricionais. Recomendações Nutricionais. Valores de Referências. Ingestão de Energia.

\section{1- INTRODUÇÃO}

A nutrição é um dos fatores determinantes para garantir crescimento e desenvolvimento adequados em crianças, favorecendo o alcance de seus potenciais genéticos. No entanto, a determinação das necessidades nutricionais é bastante complexa, tendo em vista a grande variabilidade existente entre os seres humanos, tanto em termos de características genéticas, quanto biológicas, sociais e culturais. Acredita-se que mesmo estando em uma mesma faixa etária e sexo, ainda existam variações das necessidades entre indivíduos ${ }^{1}$.
Com o propósito de auxiliar no planejamento alimentar e avaliação da ingestão de nutrientes para indivíduos saudáveis de acordo com o estágio da vida e gênero, os Comitês de Especialistas do Food and Nutrition Board, o Institute of Medicine (IOM) da National Academy of Sciences dos Estados Unidos e o Health Canadá desenvolveram extensas publicações que abordam o desenvolvimento e aplicação dos valores de referência de ingestão dietética (Dietary Reference Intakes - DRIs) para a população americana e canadense ${ }^{2}$.

Considerando a importância da avaliação do 
consumo alimentar na atenção integral à criança e a fim de subsidiar os profissionais de saúde no diagnóstico e acompanhamento nutricional, o presente artigo objetivou sistematizar informações práticas sobre as novas recomendações nutricionais e seu modo de aplicação, que foram publicadas em 8 volumes, com total de 4837 páginas. Para esta sistematização foram utilizados os relatórios das DRIs referentes a aplicação dos valores na avaliação ${ }^{3}$ e planejamento de dietas ${ }^{4}$; macrominerais, vitamina D e flúor ${ }^{5}$, energia e macronutrientes ${ }^{6}$; vitaminas hidrossolúveis ${ }^{7}$; vitaminas lipossolúveis, microminerais e elementos-traço ${ }^{8}$; vitaminas e minerais antioxidantes ${ }^{9}$ e água e eletrólitos ${ }^{10}$.

\section{2- VALORES DE REFERÊNCIA DE INGES- TÃO DIETÉTICA}

Foram propostos a partir do final da década de 90, em substituição às recomendações propostas em 1989 (RDAs) ${ }^{11}$, visando a implementação de inovações consideradas fundamentais para o aprimoramento da avaliação e planejamento da ingestão de nutrientes, como a criação de novos conceitos de avaliação e planejamento de dietas, devido à disponibilidade de informações atualizadas sobre necessidades e ingestão de nutrientes; o estabelecimento dos níveis máximos de ingestão com o surgimento do conceito de redução do risco de doenças crônico-degenerativas; e a subdivisão dentro de cada faixa etária, devido às demandas diferenciadas de nutrientes para cada etapa da vida ${ }^{1}$.

Para crianças, a divisão das faixas etárias foi estabelecida utilizando o critério biológico da maior velocidade de crescimento que ocorre durante o período de 1 a 3 anos, em relação ao ritmo observado em crianças de 4 e 5 anos. Outro aspecto considerado diz respeito à característica social do grupo, representada pela entrada das crianças na escola. As crianças de 1 a 3 anos foram mantidas na mesma faixa etária que nas RDAs de 1989 e as de 4 a 6 e de 7 a 10 anos foram reagrupadas nos grupos etários de 4 a 8 e de 9 a $13 \operatorname{anos}^{5,11}$.

A categoria de 4 a 8 anos foi estabelecida em função das maiores mudanças biológicas em relação à velocidade de crescimento e mudanças endócrinas que ocorrem neste período. Como o conhecimento atual aponta que um desenvolvimento da puberdade mais precoce, crianças de nove e dez anos foram incluídas no grupo dos adolescentes ${ }^{6}$.

\section{3- CONCEITOS}

As DRIs consistem num conjunto de quatro valores de referência de ingestão de nutrientes, que incorporam as recomendações dietéticas: necessidade média estimada (Estimated Average Requirement EAR), ingestão dietética recomendada (Recommended Dietary Allowance -RDA), ingestão adequada (Adequate Intake - AI) e limite máximo de ingestão tolerável sem que haja efeitos adversos à saúde (Tolerable Upper Intake Level -UL).

A necessidade média estimada (EAR) é o valor de ingestão diária de um nutriente que se estima para atender às necessidades de $50 \%$ de indivíduos saudáveis de um grupo em determinada faixa etária, condição fisiológica e gênero e, por definição, uma ingestão neste nível pode ser inadequada para metade do grupo. Para examinar a possibilidade de inadequação e complementar a avaliação, é necessário analisar exames bioquímicos, clínicos e antropométricos.

A ingestão adequada (AI) representa um valor de ingestão de nutriente, não de necessidade, que provavelmente excede a real necessidade de quase todos os indivíduos saudáveis, dentro de um determinado gênero e estágio de vida.

A ingestão dietética recomendada (RDA) é a quantidade do nutriente suficiente para atender à necessidade da maior parte da população, aproximadamente 97 a $98 \%$ dos indivíduos saudáveis de um grupo em determinado estágio de vida e gênero. A RDA é definida como o valor equivalente a dois desvios padrão (DP) acima da EAR (RDA = EAR + 2DP).

O limite superior tolerável de ingestão (UL) é o mais alto nível de ingestão habitual do nutriente, que provavelmente não coloca em risco de efeitos adversos, aproximadamente, todos os indivíduos de um determinado estágio de vida e gênero. Quando a ingestão aumenta acima do UL, aumenta o risco potencial de efeitos prejudiciais à saúde. Tem alta probabilidade de ser tolerado biologicamente, o que não significa que este valor seja recomendado ${ }^{3-10}$.

\section{4- USO E APLICAÇÕES}

Aplica-se a EAR para avaliar a dieta, tanto de indivíduos quanto de grupos populacionais e para calcular a RDA (Tabela I). Quando um valor de EAR para um nutriente estiver disponível, este é o valor que deve ser utilizado para fazer uma estimativa quantitativa da adequação da ingestão habitual do nutriente 
para indivíduos e grupos, uma vez que não se conhece a verdadeira necessidade dos indivíduos a serem avaliados.

Para o indivíduo, o método mais simples para avaliar a probabilidade de adequação de nutrientes é a abordagem qualitativa que recomenda que, se a ingestão do nutriente for menor que a EAR, esta deve ser implementada; se estiver entre a EAR e a RDA, existe risco de inadequação, e provavelmente a ingestão deva ser aumentada; e se estiver acima da RDA, e, ao mesmo tempo, um número expressivo de dias de consumo alimentar tiver sido avaliado, então é pouco provável que a ingestão do indivíduo seja inadequada.

Entretanto, a EAR ainda não foi estabelecida para todos os nutrientes, como para a vitamina K, cromo, manganês, cálcio, ácido pantotênico, biotina, colina, ácido linoléico, ácido linolênico, fibras, água, potássio, sódio e cloro. Para estes nutrientes, a AI é o valor de referência disponível que deve ser utilizado ${ }^{5-10}$.

Qualitativamente, avalia-se a ingestão habitual do nutriente e se este estiver acima da AI, implica que é baixa a prevalência de inadequação e se estiver abaixo da AI, nada pode ser concluído.

Na utilização do UL para verificar a suspeita de ingestão excessiva do nutriente, os profissionais devem levar em conta outros parâmetros como: fonte do nutriente, estado fisiológico e patológico do indivíduo, padrão de atividade física e período de tempo de ingestão habitual elevada do nutriente. O UL deve ser utilizado como valor limite e não como referência a ser atingida.

Para o planejamento de dietas para indivíduos utiliza-se a RDA. Quando ainda não foi determinada, é o valor de AI que deve ser utilizado. Para prevenção do excesso de nutrientes em determinado plano alimentar, utiliza-se como limite superior o UL.

Para avaliar a ingestão de nutrientes de grupos, deve ser utilizada a distribuição da ingestão no grupo e não a média, como era anteriormente empregada na metodologia da RDA de 1989 e o valor de referência a ser utilizado é a EAR. Se não houver EAR e o valor de ingestão for igual ou superior a AI, assume-se que a ingestão do grupo tem baixa prevalência de inadequação, no entanto quando a ingestão for menor que AI, nada poderá ser assumido sobre a inadequação do consumo. A distribuição da ingestão usual do grupo determina qual é a proporção de indivíduos que está acima do UL e estes podem apresentar risco de efeitos adversos à saúde.

Planejar a ingestão de nutrientes para um programa de alimentação de um grupo de pessoas é um processo muito mais complexo do que planejar para um indivíduo. Devem-se selecionar os objetivos de ingestão de nutrientes, considerando a homogeneidade ou heterogeneidade do grupo relacionada a gênero e idade, e estimar metas de distribuição da ingestão usual com EAR e UL, e se não tiver EAR, utilizar AI como mediana da distribuição.

\begin{tabular}{lcc}
\multicolumn{3}{l}{ Tabela I: Uso dos Valores de Referência de In- } \\
gestão Dietética para indivíduos e grupos. \\
\hline Objetivo & Indivíduos & Grupos \\
\hline Avaliação dietética & EAR & EAR \\
& Al & Al \\
& UL & UL \\
Planejamento dietético & RDA & EAR \\
& Al & Al \\
& UL & UL
\end{tabular}

Fonte: Adaptada de $\mathrm{IOM}^{3,4}$.

\section{5- RECOMENDAÇÕES NUTRICIONAIS}

\section{1- Energia}

As necessidades energéticas de crianças de um a dois anos incluem a quantidade necessária para o crescimento e para uma boa saúde, sendo o crescimento satisfatório um indicador sensível de que tais necessidades estão sendo atendidas. Durante o primeiro mês de vida, o percentual do gasto energético total (GET) destinado para o crescimento diminui em $35 \%$. No final de um ano, reduz em $3 \%$ e permanece baixo até o estirão da puberdade ${ }^{6}$.

A necessidade estimada de energia (estimated energy requirement - EER) é o valor médio de ingestão energética proveniente da dieta para a manutenção do balanço energético de indivíduos saudáveis. A equação da EER foi construída pela análise de dados de necessidades energéticas de crianças eutróficas obtidos pelo método da água duplamente marcada e considera o GET adicionado da quantidade de energia necessária para o depósito nos tecidos. Em crianças de 1 a 2 anos, a equação é independente do gênero, porém para meninos e meninas eutróficos com 3 a 8 anos foi estabelecida separadamente, em função das variações existentes na taxa de crescimento e no nível de atividade física (Tabela II).

Para a determinação do nível de atividade física foi utilizado o conceito de equivalentes metabóli- 
cos, sendo que as categorias de atividades foram expressas em termos de minutos de caminhada diária, numa velocidade de 2,5 milhas por hora (aproximadamente $4 \mathrm{~km} /$ hora). Com a inclusão de atividade física equivalente a 120 minutos de caminhada a $4 \mathrm{~km} / \mathrm{h}$, a criança deixa de ser considerada sedentária e passa a ser pouco ativa; com 230 minutos passa a ser ativa e 400 minutos, muito ativa.

O GET de crianças de 1 a 2 anos é constituído pelo gasto energético basal (GEB), referente ao metabolismo basal do cérebro, fígado, coração e rins; pelo efeito térmico dos alimentos, que se refere à energia gasta para a digestão, absorção, transporte e metabolismo dos nutrientes; pela termoregulação (energia gasta para manter a temperatura corporal) e pelo nível de atividade física, além de sofrer influência da idade e do gênero (maior em meninos do que em meninas).
Em crianças de 3 a 8 anos que apresentam peso normal, o efeito térmico dos alimentos corresponde a $5,9 \%$ da energia ingerida e o nível de atividade física apresenta variação em conseqüência das diferenças de estilo de vida, de hábitos geográficos e de condições sócio-econômicas entre as crianças.

Além dos cálculos de necessidade estimada de energia estabelecida para crianças com peso dentro da faixa de normalidade, foram desenvolvidos cálculos de GET para as crianças com excesso de peso, com o propósito de promover perda gradual de gordura corporal, através da manutenção do peso acompanhando o crescimento normal (Tabela III). Esta proposta se baseia na contra-indicação de uma perda de peso rápida em crianças devido aos riscos que têm de desenvolver retardo no crescimento e desenvolvimento, e deficiências de micronutrientes.

Tabela II: Necessidade estimada de energia para crianças eutróficas de 1 a 8 anos.

Crianças de 13-36m:

$\mathrm{EER}=(89 \times$ peso $[\mathrm{kg}]-100)+20$ (kcal para deposição de energia) .

Meninos de 3 a 8 anos:

$E E R=88,5-(61,9 \times$ idade $[$ anos $])+[$ FA $\times(26,7 \times$ peso $[\mathrm{kg}]+903 \times$ altura $[\mathrm{m}])]+$ 20 (kcal para deposição de energia).

Meninas de 3 a 8 anos: $E E R=135,3-(30,8 \times$ idade $[$ anos $])+[F A \times(10,0 \times$ peso $[\mathrm{kg}]+934 \times$ altura $[\mathrm{m}])]+$ 20 (kcal para deposição energética)
FATOR ATIVIDADE (FA):

Sedentários: 1,0

Pouco ativos:

1,13 (meninos)

1,16 (meninas)

Ativos:

1,26 (meninos)

1,31 (meninas)

Muito ativos:

1,42 (meninos)

1,56 (meninas)

Fonte: Adaptada de $\mathrm{IOM}^{6}$.

Tabela III: Necessidade estimada de energia para crianças com excesso de peso de $\mathbf{3}$ a 8 anos.

\begin{tabular}{|c|c|}
\hline Meninos de 3 a 8 anos: & $\begin{array}{l}\text { FATOR ATIVIDADE (FA): } \\
\text { Sedentários: } \\
1.0\end{array}$ \\
\hline GET $=114-(50,9 \times$ idade $[$ anos $])+[$ FA $\times(19,5 \times$ peso $[\mathrm{kg}]+1161,4 \times$ altura $[\mathrm{m}])]$ & $\begin{array}{l}\text { Pouco ativos: } \\
\text { 1,12 (meninos) } \\
1,18 \text { (meninas) }\end{array}$ \\
\hline $\begin{array}{l}\text { Meninas de } 3 \text { a } 8 \text { anos: } \\
\text { GET }=389-(41,2 \times \text { idade }[\text { anos }])+[\text { FA } \times(15,0 \times \text { peso }[\mathrm{kg}]+701,6 \times \text { altura }[\mathrm{m}])]\end{array}$ & $\begin{array}{c}\text { Ativos: } \\
1,24 \text { (meninos) } \\
\text { 1,35 (meninas) } \\
\text { Muito ativos: } \\
1,45 \text { (meninos) } \\
1,60 \text { (meninas) }\end{array}$ \\
\hline
\end{tabular}

Fonte: Adaptada de $\mathrm{IOM}^{6}$. 


\section{1- Macronutrientes}

A determinação dos valores de referência para os macronutrientes ${ }^{6}$ estão descritos abaixo e as recomendações estão apresentadas na Tabela IV.

\subsection{1- Carboidratos}

A recomendação de carboidratos se baseia na quantidade mínima de glicose necessária para suprir as necessidades do cérebro. $\mathrm{O}$ consumo de glicose pelo cérebro depois de 1 ano de idade permanece constante ou aumenta modestamente, e está dentro da faixa apresentada para adultos (aproximadamente $31 \mu \mathrm{mol} / 100 \mathrm{~g}$ de cérebro/minuto). Portanto, é provável que crianças de 2 a 9 anos apresentem necessidade similar à de adultos para carboidratos.

A EAR e RDA foram determinadas por meio de extrapolações dos valores referentes ao adulto e não varia entre os gêneros, uma vez que as diferenças na utilização de glicose pelo cérebro são pequenas. A faixa de distribuição aceitável de carboidratos para crianças também é a mesma dos adultos, de 45 a $65 \%$ do total de ingestão energética diária.

Admitindo um consumo de energia compatível com as necessidades estimadas, é sugerido que um máximo de $25 \%$ da energia seja proveniente de açúcar adicionado, uma vez que um aumento neste consumo pode elevar o risco de inadequação da ingestão de micronutrientes. A ingestão deste açúcar pode ser diminuída pela inclusão de açúcares naturalmente presentes em alimentos como os do leite, de produtos lácteos e de frutas, que inclusive são alimentos ricos em micronutrientes, e pela restrição do consumo de bebidas açucaradas, refrigerantes e doces.

\subsection{2- Proteínas}

A EAR foi estimada pelo método fatorial, que combina a quantidade necessária para a manutenção baseada no peso corporal (equilíbrio nitrogenado) e a quantidade estimada necessária para deposição de proteínas. A faixa de distribuição aceitável de proteína é de 5 a $20 \%$ do total energético diário para crianças de 1 a 3 anos e 10 a $30 \%$ para 4 a 18 anos.

\subsection{3- Gorduras e ácidos graxos}

As DRIs para gorduras não puderam ser estabelecidas pela falta de dados, porém houve a determinação da faixa de distribuição aceitável que propõe que a porcentagem de energia proveniente de gordura seja de 30 a $40 \%$ para crianças de 1 a 3 anos e de 25 a $35 \%$ entre 4 a 18 anos. Vale destacar que dietas com baixo conteúdo de gordura podem levar a redução da ingestão de alguns micronutrientes e as com grandes quantidades podem acarretar em um risco potencial de obesidade.

Para o ácido linoléico e o ácido linolênico, a AI foi estabelecida com base no consumo médio de indivíduos americanos, em que a presença da deficiência destes ácidos graxos praticamente não existe.

\subsection{4- Fibras}

A recomendação de consumo de fibras para crianças considerou a premissa de que, assim como para os adultos, um consumo adequado deve proporcionar funcionamento normal do intestino, prevenir câncer relacionado à dieta e diminuir a concentração sérica de colesterol para redução do risco de doença cardiovascular e prevenção da obesidade.

Tabela IV: Valores de referência das recomendações diárias (DRIs) de macronutrientes para crianças de 1 a 8 anos.

\begin{tabular}{rcrrrr}
\hline Nutriente & Idade (anos) & EAR & RDA & Al & UL \\
\hline Carboidratos & $1-3$ & $100 \mathrm{~g}$ & $130 \mathrm{~g}$ & - & - \\
& $4-8$ & $100 \mathrm{~g}$ & $130 \mathrm{~g}$ & - & - \\
Proteínas & $1-3$ & $0,87 \mathrm{~g} / \mathrm{kg}$ & $1,05 \mathrm{~g} / \mathrm{kg}$ & - & - \\
& $4-8$ & $0,76 \mathrm{~g} / \mathrm{kg}$ & $0,95 \mathrm{~g} / \mathrm{kg}$ & - & - \\
Ácido Linoléico & $1-3$ & - & - & $7 \mathrm{~g}$ & - \\
& $4-8$ & - & - & $10 \mathrm{~g}$ & - \\
Ácido Linolênico & $1-3$ & - & - & $0,7 \mathrm{~g}$ & - \\
& $4-8$ & - & - & $0,9 \mathrm{~g}$ & - \\
Fibras & $1-3$ & - & - & $19 \mathrm{~g}$ & - \\
& $4-8$ & - & - & $25 \mathrm{~g}$ & - \\
\hline
\end{tabular}

Fonte: Adaptada de $\mathrm{IOM}^{6}$. 
A AI para fibras totais foi baseada nos valores utilizados para adultos, que apontam que $14 \mathrm{~g} / 1000 \mathrm{kcal}$ reduz o risco de doença cardiovascular. Foi estabelecida AI de 19g/dia de fibra total para crianças de 1 a 3 anos, proporcional a média de energia ingerida (1372kcal) e de $25 \mathrm{~g} /$ dia para crianças de 4 e 8 anos, cuja média de ingestão energética é de $1759 \mathrm{kcal} / \mathrm{dia}$.

Devido à relação existente entre consumo de fibras e energia, crianças que consomem uma quantidade de energia menor que a estimada para cada categoria etária, necessitam de uma ingestão de fibras menor que a recomendada pela AI.

\section{2- Água e eletrólitos}

A determinação dos valores de referência para água e eletrólitos ${ }^{10}$ estão descritos a seguir e as recomendações estão apresentadas na Tabela V.

\subsection{1- Água}

Como não existe um nível de ingestão de água recomendado para alcançar uma hidratação adequada e uma saúde ótima, utilizou-se a média de ingestão total encontrada em um estudo americano, de 1,3L/d e 1,7L/d (respectivamente em crianças de 1 a 3 anos e de 4 a 8 anos), para estabelecer o valor de AI para água total. Como a porcentagem de água total presente nos alimentos consumidos foi de $29 \%$ para crianças de 1 a 3 anos (0,38L/d) e $29 \%$ para crianças de 4 a 8 anos $(0,51 \mathrm{~L} / \mathrm{d})$, a ingestão de bebidas, incluindo água, necessária para alcançar os valores de AI, deve totalizar $0,9 \mathrm{~L} / \mathrm{d}$ (4 copos) para crianças de 1 a 3 anos e 1,2L/d (5 copos) para as de 4 a 8 anos.

\subsection{1- Potássio}

A pressão sanguínea é um indicador potente das necessidades de potássio, porém poucos estudos têm avaliado a relação entre a ingestão de potássio e a pressão sanguínea ou seu aumento durante a infância. Devido às condições resultantes da deficiência de potássio (elevada pressão sanguínea, desmineralização óssea e pedras nos rins) serem crônicas e provavelmente resultarem de uma ingestão deficiente em um período prolongado, incluindo a infância, é adequado extrapolar as recomendações de ingestão de potássio de adultos para crianças, ajustando-as para uma menor ingestão energética que é observada nesta faixa etária.

\subsection{1- Sódio e Cloro}

Como a maturação dos rins é similar entre crianças e adultos, não há razões para considerar que as necessidades de sódio sejam diferentes entre crianças e adultos. Observa-se que crianças têm a habilidade de conservar o sódio no corpo na ocorrência de baixos níveis de ingestão dietética. A AI foi estabelecida por extrapolações dos valores dos adultos, ajustando-a para a ingestão de energia. O cloro estará adequado quando consumido em quantidade equimolar ao sódio.

$\mathrm{O}$ valor médio de ingestão estimada de energia no adulto é de $2150 \mathrm{kcal}$ e a AI de sódio é de $1,5 \mathrm{~g} /$ dia. Considerando que o valor de ingestão média de energia para crianças de 1 a 3 anos é de $1372 \mathrm{kcal}$, a AI é de $1 \mathrm{~g} / \mathrm{dia}(1372 \mathrm{kcal} / 2150 \mathrm{kcal} \times 1,5 \mathrm{~g} / \mathrm{dia})$ e para crianças de 4 a 8 anos, a AI é de 1,2g/dia (1759kcal/2150kcal $\mathrm{x} 1,5 \mathrm{~g} / \mathrm{dia})$.

Tabela V: Valores de referência das recomendações diárias (DRIs) de água e eletrólitos para crianças de 1 a 8 anos.

\begin{tabular}{rccccc}
\hline Nutriente & Idade (anos) & EAR & RDA & Al & UL \\
\hline Água & $1-3$ & - & - & $1,3 \mathrm{~L}$ & - \\
& $4-8$ & - & - & $1,7 \mathrm{~L}$ & - \\
Potássio & $1-3$ & - & - & $3,0 \mathrm{~g}$ & - \\
Sódio & $4-8$ & - & - & $1,0 \mathrm{~g}$ & $1,5 \mathrm{~g}$ \\
Cloro & $1-3$ & - & - & $1,2 \mathrm{~g}$ & $1,9 \mathrm{~g}$ \\
& $4-8$ & - & - & $1,5 \mathrm{~g}$ & $2,3 \mathrm{~g}$ \\
\hline
\end{tabular}

Fonte: Adaptada de $\mathrm{IOM}^{10}$. 


\section{3- Micronutrientes}

Os valores de referência das DRIs de Vitamina C, Vitamina E, Selênio, Vitamina A, Vitamina K, Cromo, Cobre, Iodo, Manganês, Molibdênio, Zinco, Magnésio, Flúor, Tiamina, Riboflavina, Niacina, Vitamina $\mathrm{B}_{6}$, Folato, Vitamina $\mathrm{B}_{12}$, Ácido Pantotênico, Biotina e Colina foram estabelecidos através de extrapolações das necessidades do adulto e estão apresentados na Tabela VI. As fórmulas apresentadas a seguir foram utilizadas para estabelecer a EAR destes nutrientes, para estas faixas etárias.
$\mathrm{EAR}_{\text {crianças }}=\mathrm{EAR}_{\text {adultos }} \times \mathrm{F}$

Onde $\mathrm{F}=\left(\mathrm{Peso}_{\text {criança }} / \mathrm{Peso}\right.$ adulto $^{0,75} \mathrm{x}(1+$ Fator de crescimento)

Fator de crescimento $=0,3$ (7 meses a 3 anos); 0,15 (4 a 8 anos).

Quando a EAR não estiver disponível, utilizase a AI de adultos na fórmula acima.

Tabela VI: Valores de referência das recomendações diárias (DRIs) de micronutrientes para crianças de 1 a 8 anos.

\begin{tabular}{|c|c|c|c|c|c|}
\hline Nutriente & Idade & EAR & RDA & $\mathrm{Al}$ & UL \\
\hline \multirow[t]{2}{*}{ Vitamina C } & $1-3$ & $13 \mathrm{mg}$ & $15 \mathrm{mg}$ & - & $400 \mathrm{mg}$ \\
\hline & $4-8$ & $22 \mathrm{mg}$ & $25 \mathrm{mg}$ & - & $650 \mathrm{mg}$ \\
\hline \multirow[t]{2}{*}{ Vitamina E } & $1-3$ & $5 \mathrm{mg}$ & $6 \mathrm{mg}$ & - & $200 \mathrm{mg}$ \\
\hline & $4-8$ & $6 \mathrm{mg}$ & $7 \mathrm{mg}$ & - & $300 \mathrm{mg}$ \\
\hline \multirow[t]{2}{*}{ Selênio } & $1-3$ & $17 \mu \mathrm{g}$ & $20 \mu \mathrm{g}$ & - & $90 \mu \mathrm{g}$ \\
\hline & $4-8$ & $23 \mu \mathrm{g}$ & $30 \mu \mathrm{g}$ & - & $150 \mu \mathrm{g}$ \\
\hline \multirow[t]{2}{*}{ Vitamina $A^{a}$} & $1-3$ & $210 \mu \mathrm{g}$ & $300 \mu \mathrm{g}$ & - & $600 \mu \mathrm{g}$ \\
\hline & $4-8$ & $275 \mu \mathrm{g}$ & $400 \mu \mathrm{g}$ & - & $900 \mu \mathrm{g}$ \\
\hline \multirow[t]{2}{*}{ Vitamina K } & $1-3$ & - & - & $30 \mu \mathrm{g}$ & - \\
\hline & $4-8$ & - & - & $55 \mu \mathrm{g}$ & - \\
\hline \multirow[t]{2}{*}{ Cromo } & $1-3$ & - & - & $11 \mu \mathrm{g}$ & - \\
\hline & $4-8$ & - & - & $15 \mu \mathrm{g}$ & - \\
\hline \multirow[t]{2}{*}{ Cobre } & $1-3$ & $260 \mu \mathrm{g}$ & $340 \mu \mathrm{g}$ & - & $1 \mathrm{mg}$ \\
\hline & $4-8$ & $340 \mu \mathrm{g}$ & $440 \mu \mathrm{g}$ & - & $3 \mathrm{mg}$ \\
\hline \multirow[t]{2}{*}{ lodo } & $1-3$ & $65 \mu \mathrm{g}$ & $90 \mu \mathrm{g}$ & - & $200 \mu \mathrm{g}$ \\
\hline & $4-8$ & $65 \mu \mathrm{g}$ & $90 \mu \mathrm{g}$ & - & $300 \mu \mathrm{g}$ \\
\hline \multirow[t]{2}{*}{ Ferro } & $1-3$ & $3,0 \mathrm{mg}$ & $7 \mathrm{mg}$ & - & $40 \mathrm{mg}$ \\
\hline & $4-8$ & $4,1 \mathrm{mg}$ & $10 \mathrm{mg}$ & - & $40 \mathrm{mg}$ \\
\hline \multirow[t]{2}{*}{ Manganês } & $1-3$ & - & - & $1,2 \mathrm{mg}$ & $2 \mathrm{mg}$ \\
\hline & $4-8$ & - & - & $1,5 \mathrm{mg}$ & $3 \mathrm{mg}$ \\
\hline \multirow[t]{2}{*}{ Molibdênio } & $1-3$ & $13 \mu \mathrm{g}$ & $17 \mu \mathrm{g}$ & - & $0,3 \mathrm{mg}$ \\
\hline & $4-8$ & $17 \mu \mathrm{g}$ & $22 \mu \mathrm{g}$ & - & $0,6 \mathrm{mg}$ \\
\hline
\end{tabular}




\begin{tabular}{|c|c|c|c|c|c|}
\hline \multirow[t]{2}{*}{ Zinco } & $1-3$ & $2,5 \mathrm{mg}$ & $3 \mathrm{mg}$ & - & $7 \mathrm{mg}$ \\
\hline & $4-8$ & $4 \mathrm{mg}$ & $5 \mathrm{mg}$ & - & $12 \mathrm{mg}$ \\
\hline \multirow[t]{2}{*}{ Cálcio } & $1-3$ & - & - & $500 \mathrm{mg}$ & $2500 \mathrm{mg}$ \\
\hline & $4-8$ & - & - & $800 \mathrm{mg}$ & $2500 \mathrm{mg}$ \\
\hline \multirow[t]{2}{*}{ Fósforo } & $1-3$ & $380 \mathrm{mg}$ & $460 \mathrm{mg}$ & - & $3 g$ \\
\hline & $4-8$ & $405 \mathrm{mg}$ & $500 \mathrm{mg}$ & - & $3 g$ \\
\hline \multirow[t]{2}{*}{ Magnésio $^{b}$} & $1-3$ & $65 \mathrm{mg}$ & $80 \mathrm{mg}$ & - & $65 \mathrm{mg}$ \\
\hline & $4-8$ & $110 \mathrm{mg}$ & $130 \mathrm{mg}$ & - & $110 \mathrm{mg}$ \\
\hline \multirow[t]{2}{*}{ Vitamina D } & $1-3$ & - & - & $5 \mu \mathrm{g}$ & $50 \mu \mathrm{g}$ \\
\hline & $4-8$ & - & - & $5 \mu \mathrm{g}$ & $50 \mu \mathrm{g}$ \\
\hline \multirow[t]{2}{*}{ Fluor } & $1-3$ & - & - & $0,7 \mathrm{mg}$ & $1,3 \mathrm{mg}$ \\
\hline & $4-8$ & - & - & $1 \mathrm{mg}$ & $2,2 \mathrm{mg}$ \\
\hline \multirow[t]{2}{*}{ Tiamina } & $1-3$ & $0,4 \mathrm{mg}$ & $0,5 \mathrm{mg}$ & - & - \\
\hline & $4-8$ & $0,5 \mathrm{mg}$ & $0,6 \mathrm{mg}$ & - & - \\
\hline \multirow[t]{2}{*}{ Riboflavina } & $1-3$ & $0,4 \mathrm{mg}$ & $0,5 \mathrm{mg}$ & - & - \\
\hline & $4-8$ & $0,5 \mathrm{mg}$ & $0,6 \mathrm{mg}$ & - & - \\
\hline \multirow[t]{2}{*}{ Niacinab } & $1-3$ & $5 \mathrm{mg}$ & $6 \mathrm{mg}$ & - & $10 \mathrm{mg}$ \\
\hline & $4-8$ & $6 \mathrm{mg}$ & $8 \mathrm{mg}$ & - & $15 \mathrm{mg}$ \\
\hline \multirow[t]{2}{*}{ Vitamina $B_{6}$} & $1-3$ & $0,4 \mathrm{mg}$ & $0,5 \mathrm{mg}$ & - & $30 \mathrm{mg}$ \\
\hline & $4-8$ & $0,5 \mathrm{mg}$ & $0,6 \mathrm{mg}$ & - & $40 \mathrm{mg}$ \\
\hline \multirow[t]{2}{*}{ Folato $^{C}$} & $1-3$ & $120 \mu \mathrm{g}$ & $150 \mu \mathrm{g}$ & - & $300 \mu \mathrm{g}$ \\
\hline & $4-8$ & $160 \mu \mathrm{g}$ & $200 \mu \mathrm{g}$ & - & $400 \mu \mathrm{g}$ \\
\hline \multirow[t]{2}{*}{ Vitamina $B_{12}$} & $1-3$ & $0,7 \mu \mathrm{g}$ & $0,9 \mu \mathrm{g}$ & - & - \\
\hline & $4-8$ & $1,0 \mu \mathrm{g}$ & $1,2 \mu \mathrm{g}$ & - & - \\
\hline \multirow[t]{2}{*}{ Ácido Pantotênico } & $1-3$ & - & - & $2 \mathrm{mg}$ & - \\
\hline & $4-8$ & - & - & $3 \mathrm{mg}$ & - \\
\hline \multirow[t]{2}{*}{ Biotina } & $1-3$ & - & - & $8 \mu \mathrm{g}$ & - \\
\hline & $4-8$ & - & - & $12 \mu \mathrm{g}$ & - \\
\hline \multirow[t]{2}{*}{ Colina } & $1-3$ & - & - & $200 \mathrm{mg}$ & $1 \mathrm{~g}$ \\
\hline & $4-8$ & - & - & $250 \mathrm{mg}$ & $1 \mathrm{~g}$ \\
\hline
\end{tabular}

Fonte: Adaptada de $\mathrm{IOM}^{5,7 / 9}$.

${ }^{a} A$ unidade para a vitamina $A$ é $\mu \mathrm{g}$ de equivalentes da atividade do retinol (RAE) que corresponde a 1 RAE $=1 \mu \mathrm{g}$ de retinol ou $12 \mu \mathrm{g}$ de $\beta$-caroteno ou $24 \mu \mathrm{g}$ de outros carotenóides pró-vitamina $\mathrm{A}^{2 . \mathrm{b}} \mathrm{O}$ UL de magnésio considera apenas agentes farmacológicos, não alimentos ${ }^{9}$; o UL de niacina considera suplementos, alimentos fortificados e agentes farmacológicos. ${ }^{\mathrm{C}} \mathrm{A}$ unidade para o folato é $\mu \mathrm{g}$ de equivalentes de folato dietético (DFE) que corresponde a DFE $=\mu \mathrm{g}$ de folato dietético $+(1,7 \times \mu \mathrm{g}$ de ácido fólico sintético), assumindo-se uma biodisponibilidade de ácido fólico adicionado aos alimentos de $85 \%$ e do folato dietético de $50 \%$ $(1,7 \text { vez maior })^{2}$. 
As DRIs de Ferro ${ }^{8}$, Cálcio, Fósforo e Vitamina $\mathrm{D}^{5}$ foram estabelecidas utilizando dados de estudos e considerações que estão descritos abaixo.

\subsection{1- Ferro}

A necessidade média estimada de ferro (EAR) foi determinada por um modelo fatorial baseado nos componentes da necessidade, tais como perdas basais de ferro, aumento da massa de hemoglobina, aumento nos tecidos (ferro não estocado) e aumento nos estoques de ferro, considerando uma absorção máxima de $18 \%$. Vale destacar que a taxa de crescimento corporal exerce uma influência fundamental no aumento das necessidades deste mineral.

\subsection{2- Cálcio}

Um valor preciso de ingestão média necessária para promover a assimilação de cálcio não pôde ser obtido pelos dados disponíveis até o presente momento. Os estudos de balanço de cálcio em meninas de 4 a 8 anos indicaram que uma ingestão entre 800 e 900 $\mathrm{mg} / \mathrm{d}$ pode implicar em uma retenção acima de 174 $\mathrm{mg} / \mathrm{d}$, razão pela qual a AI foi estabelecida em 800 $\mathrm{mg} / \mathrm{d}$ para esta faixa etária. Como não há estudos disponíveis avaliando meninos, os dados das meninas devem ser utilizados para ambos os sexos.

Para estabelecer a AI de crianças entre 1 a 3 anos de vida considerou-se que a retenção de cálcio durante este estágio de vida é de aproximadamente $100 \mathrm{mg} / \mathrm{d}$ e há a estimativa de que ocorre $20 \%$ de retenção de cálcio ingerido. Tendo por base os dados de crianças de 4 a 8 anos, foi estabelecida uma AI de $500 \mathrm{mg} / \mathrm{d}$ para as de 1 a 3 anos.

\subsection{3- Fósforo}

A EAR proverá as necessidades fisiológicas para a retenção de fósforo na massa magra e óssea, considerando as perdas urinárias da quantidade total ingerida. Devido ao fato da excreção urinária aumentar linearmente com a ingestão dietética deste mineral, não parece apropriado acrescentar à EAR uma quantidade que exceda as necessidades fisiológicas para crescimento e manutenção.

\subsection{4- Vitamina D}

Não há dados disponíveis para propor um valor com a quantidade necessária de vitamina $\mathrm{D}$ para prevenir deficiência em crianças de 1 a 8 anos. Dados de crianças um pouco mais velhas e de crianças de diferentes continentes que não são expostas aos raios solares mostram que a maioria das crianças que têm uma

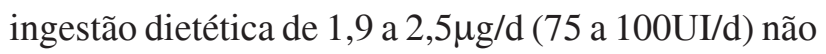
apresentam evidência de deficiência e têm valores séricos normais de $25(\mathrm{OH}) \mathrm{D}$. Para cobrir as necessidades da maioria das crianças de 1 a 8 anos, independente da exposição ao sol, o valor acima é dobrado para uma AI de $5 \mu \mathrm{g} / \mathrm{d}(200 \mathrm{UI} / \mathrm{d})$.

\section{6- CONSIDERAÇÕES FINAIS}

A compreensão do modo como foram estabelecidas as DRIs evidencia a necessidade de novos estudos que apresentem informações consistentes sobre as necessidades nutricionais da faixa etária estudada, visto que os valores para Vitamina C, Vitamina E, Selênio, Vitamina A, Vitamina K, Cromo, Cobre, Iodo, Manganês, Molibdênio, Zinco, Magnésio, Flúor, Tiamina, Riboflavina, Niacina, Vitamina $\mathrm{B}_{6}$, Folato, Vitamina $\mathrm{B}_{12}$, Ácido Pantotênico, Biotina e Colina foram estabelecidos através de extrapolações das necessidades do adulto. Além disso, deve-se considerar que para alguns nutrientes ainda não foram estabelecidas as necessidades, mas apenas recomendações baseadas em estudos da ingestão de nutrientes em determinada população, caracterizado pelas AIs. Como exemplo podemos citar o ácido linoléico, ácido linolênico e a água.

Para o carboidrato, é de fundamental importância salientar que os valores propostos estão relacionados à quantidade mínima deste nutriente a ser ingerido para atingir as necessidades cerebrais e que os valores encontrados na prática poderão estar em níveis mais elevados do que o recomendado, sem que isto simbolize inadequação nutricional.

O novo conceito das DRIs surge com um novo parâmetro a ser constantemente considerado para o planejamento e avaliação de dietas, o UL, que protege o indivíduo ou grupo de indivíduos de uma ingestão de nutrientes em níveis lesivos à saúde, seja na forma de alimentos ou de suplementos alimentares e medicamentos.

Esta metodologia pressupõe que se conheça a população estudada, ou seja, que os parâmetros (variações de ingestão e necessidades) sejam locais. Como não dispomos destes estudos no Brasil, nos resta a utilização de parâmetros de referência da população americana e canadense, porém com análise criteriosa dos dados.

Tendo em vista estas considerações finais, constata-se a fundamental importância da produção do conhecimento relacionada às necessidades e recomendações nutricionais e aponta-se, então, a necessidade do desenvolvimento de novos estudos nesta área. 
Vieira MNCM, Japur CC, Resende CMM, Monteiro JP. Dietary reference intakes for the evaluation and planning of diets for children aged one to eight years. Medicina (Ribeirão Preto) 2008; 41 (1): 67-76.

ABSTRACT: Dietary Reference Intakes (DRIs) have been developed in order to help dietary planning and the assessment of nutrient intake for individuals and populations. These values were proposed in the late nineties to replace the recommended daily allowances (RDAs), innovating and creating new concepts of dietary planning and assessment due to the recently updated information about nutrient requirements, the establishment of maximum intake levels in need to include the concept of risk-reduction of chronic-degenerative diseases, and a subdivision within each age range due to the different nutrient requirements in each stage of life. Considering the food consumption assessment important in full-range attention to the children's health, and enabling funds to health professionals in nutritional monitoring and diagnosis, the main objective of the current study was to present, in a practice way, the nutritional recommendations and their applications for children aged 1 to 8 years old, based on the DRIs Methodology.

Keywords: Child Nutrition. Nutritional Requirements. Nutrition Policy. Reference Values. Energy Intake.

\section{REFERÊNCIAS}

1 - Amâncio OMS. Novos conceitos das Recomendações de Nutrientes. Cad Nutr 1999;18:55-8.

2 - Gebhardt SE, Holden JM. Consequences of changes in the dietary reference intakes for nutrient databases. J Food Composit Anal 2006;19:S91-5.

3 - Institute of Medicine. Dietary Reference Intakes: Applications in dietary assessment. Washington, DC: National Academic Press; 2000.

4 - Institute of Medicine. Dietary Reference Intakes: Applications in dietary planning. Washington, DC: National Academic Press; 2003.

5 - Institute of Medicine. Dietary References Intakes for Calcium, Phosphorus, Magnesium, Vitamin D and Fluoride. Washington, DC: National Academic Press; 1997.

6 - Institute of Medicine. Dietary References Intakes for Energy, Carbohydrate, Fiber, Fat, Fatty acids, Cholesterol, Protein and Amino acids. Washington, DC: National Academic Press; 2005.
7 - Institute of Medicine. Dietary References Intakes for Thiamin, Riboflavin, Niacin, Vitamin B6, Folate, Vitamin B12, Pantothenic acid, Biotin and Choline. Washington, DC: National Academic Press; 1998.

8 - Institute of Medicine. Dietary References Intakes for Vitamin A, Vitamin K, Arsenic, Boron, Chromium, Copper, lodine, Iron, Manganese, Molybdenum, Nickel, Silicon, Vanadium and Zinc. Washington, DC: National Academic Press; 2000.

9 - Institute of Medicine. Dietary References Intakes for Vitamin C, Vitamin E, Selenium and Carotenoids. Washington, DC: National Academic Press; 2000.

10 - Institute of Medicine. Dietary References Intakes for Water, Potassium, Sodium, Chloride, and Sulfate. Washington, DC: National Academic Press; 2004.

11 - Monsen ER. The 10th edition of the Recommended Dietary Allowances: what's new in the 1989 RDAs? J Am Diet Assoc 1989;89:1748-52.

Recebido para publicação em 15/06/2007.

Aprovado para publicação em 22/10/2007. 